\title{
Intermediate Weighted Fast Spin Echo (IW FSE) MR Imaging of Hyaline Cartilage Defects of the Knee: Comparison with the Fat Suppressed Three Dimensional Gradient Echo (3D SPGR) Imaging and Arthroscopy
}

\author{
Raja Kollu ${ }^{1}$, Charudutt Sambhaji ${ }^{2}$, Ramprakash HV ${ }^{3}$ \\ ${ }^{1}$ Assistant Professor, ${ }^{2}$ Associate Professor, ${ }^{3}$ Professor, Department of Radiodiagnosis, Kasturba Medical College, Manipal, \\ India
}

Corresponding author: Dr Raja Kollu, Assistant Professor, Department of Radiodiagnosis, VIMS \& RC, \# 82, EPIP Area, Whitefield, Bangalore - 560066, India

DOI: 10.21276/ijcmsr.2018.3.3.2

How to cite this article: Raja Kollu, Charudutt Sambhaji, Ramprakash HV. Intermediate weighted fast spin echo (IW FSE) MR imaging of hyaline cartilage defects of the knee: comparison with the fat suppressed three dimensional gradient echo (3D SPGR) imaging and arthroscopy. International Journal of Contemporary Medicine Surgery and Radiology. 2018;3(3):C6-C10.

\section{A B S T R A C T}

Introduction: Magnetic resonance imaging (MRI) of the knee joint is used routinely for the detection of traumatic lesions. The sensitivity of Intermediate weighted fast spin echo (IW FSE) images were compared with that of standard fat-suppressed three-dimensional spoiled gradient-echo (SPGR) MR images for detecting hyaline cartilage defects of the knee, using arthroscopy as the standard of reference.

Materials and methods: We assessed 90 consecutive patients for hyaline cartilage defects of the knee with both of Intermediate weighted fast spin echo (Iw FSE) and a sagittal fat-suppressed three-dimensional SPGR sequences. Of these patients, 50 with meniscal or ligament injury, or persistent symptoms, underwent subsequent arthroscopy. The images were then retrospectively analyzed for articular defects in a blinded fashion by two independent observers. Sensitivity, specificity, accuracy and intra observer and inter observer agreement were determined for the different imaging techniques.

Results: A total of 300 surfaces of 50 knees were evaluated, of which 221( $72 \%)$ were normal surfaces and 79 (27\%) showed cartilage defects according to arthroscopy. When looking at all surfaces combined for each reader, the Iw FSE and SPGR imaging sequences had almost equal sensitivity for detecting hyaline cartilage defects.We found no difference in specificity and accuracy ( $98 \%$ versus $98 \%, p>.87$ and $88-94 \%$ versus $88-98 \%$ ). We also found that combined evaluation of Iw FSE MR and 3D-SPGR images gave no added diagnostic advantage (sensitivity, 86\%; specificity, 98\%; $p>.48$ ). The study achieved excellent reproducibility among readings and between readers for all the surfaces of knee.

Conclusion: Intermediate weighted fast spin echo (Iw FSE) has equal sensitivity that of Fat-suppressed three-dimensional SPGR imaging for the detection of hyaline cartilage defects of the knee.

Key words: Intermediate weighted fast spin-echo (Iw FSE), 3-dimensional spoiled gradient-echo (3D SPGR)

\section{INTRODUCTION}

Traumatic cartilage lesions of the knee are common in physically active individuals, however the accurate numbers and localizations are not well known ${ }^{1,2}$.

The most crucial prerequisite for adequate long-term diarthrodial joint function is the integrity of its articular cartilage. Many previous studies ${ }^{1-4}$ have demonstrated that the prevalence of articular chondral trauma is high and cartilage damage is generally acknowledged as an early factor in the process of irreversible joint degeneration. The recent development of new surgical procedures and tissue engineering, such as autologous chondrocyte implantation (ACI), have promising results for repair of hyaline or hyaline like cartilage.
A variety of magnetic resonance imaging (MRI) sequences for assessment of articular cartilage have attracted immense interest and has been the subject of numerous research studies over the past years ${ }^{2,3}$.

With conventional spin echo sequences, sensitivities as low as $29 \%$ to $53 \%$ have been reported for the detection of hyaline cartilage lesions ${ }^{2,3}$. Better results have been published with the use of cartilage-specific sequences,like Intermediate weighted fast spin-echo sequences (Iw FSE) 4,5 , and 3-dimensional spoiled gradient-echo sequences (3DSPGR) sequences ${ }^{2,6,7}$

In this study, we have attempted to compare cartilage sensitive MRI sequences in the morphologic assessment of articular cartilage in patients with multi ligamentous injury 
to the knees by using arthroscopy as the reference standard.

\section{MATERIAL AND METHODS}

This was a prospective study conducted in the department of Radio diagnosis and imaging, Kasturba Medical College, Manipal from Oct 2010 to Aug 2012.

The study protocol was approved by our Institutional ethics committee and informed consent was obtained from all patients before the MRI study.

All patients with traumatic knee injury referred for MR evaluation were scanned on a $1.5 \mathrm{~T}$ GE HDxt MRI system using a dedicated quadrature phased array knee coil with the routine MR imaging protocol, which included 3 plane PD weighted FS. A quick preliminary assessment of the menisci and cruciate ligaments was performed after the routine protocol. Patients having multiligamentous injuries were further scanned with cartilage specific sequences and were included in the study.

Cartilage specific sequences used in our study were 3 plane Intermediate weighted (Iw-FSE) non fat sat and Sagittal three dimensional spoiled gradient echo (3D SPGR) fat sat sequences.

\section{Inclusion criteria}

1. Patients with definite history of external injury to the knee.

2. Knees with multi ligamentous injury.

\section{Exclusion criteria}

1. Prior h/o knee surgery.

2. Contraindications for MR imaging.

3. Knees with isolated meniscal or cruciate ligament injury.

4. Non availability of arthroscopy record.

\section{Image analysis}

All 300 surfaces of 50 knees were analyzed for cartilage injury. The following six articular surfaces were evaluated:

1. Medial patellar facet

2. Lateral patellar facet

3. Medial femoral condyle

4. Medial tibial condyle

5. Lateral femoral condyle

6. Lateral tibial condyle

The knees were assessed for cartilage signal and morphology, meniscal integrity, synovitis/effusion, intra-articular loose bodies, periarticular cysts, bursitis and anterior and posterior cruciate ligament integrity.

Cartilage injuries were assessed using a modified version of the Noyes classification system for articular cartilage defects:

Grade 0 Intact cartilage with normal signal and uniform thickness.

Grade 1 Focal abnormal signal without surface abnormalities.

Grade 2 Superficial ulceration or fissuring, with a depth of not more than $50 \%$ of cartilage thickness.

Grade 3 Deep ulceration or fissuring of more than $50 \%$ but less than $100 \%$ of cartilage thickness.

Grade 4 Full-thickness cartilage defect with normal or erosion of subchondral bone.

\section{STATISTICAL ANALYSIS}

Descriptive analysis, cross tabulation bar charts and grading tables were obtained using the SPSS software.

The distribution of grading of articular lesions for different articular surfaces were calculated and compared with arthroscopic grading. More than $85 \%$ of lesions showed a maximum difference of one grade between arthroscopy and MR imaging for different articular surfaces.

\section{STATISTICAL ANALYSIS}

Correlation of grades between arthroscopy and MR imaging for different articular surfaces were calculated by using spearman's rho correlation (r). Spearman's rho correlation ranges from -1 to +1 .

-1 - Negative correlation

0 - No correlation

+1 - Positive correlation

The more positive the $r$, stronger is the correlation.

\section{RESULTS}

The study group consists of 50 subjects, of whom 38 cases had history of RTA, 6 cases had history of fall and the remaining 6 cases had a history of blunt trauma. All patients had injuries in the last six months prior to the MRI examination.43

\begin{tabular}{|l|c|c|}
\hline & Iw FSE & 3DSPGR \\
\hline TR & 4000 & $\min$ \\
\hline TE & 34 & $\min$ \\
\hline FLIP ANGLE & 90 & 10 \\
\hline VBW & $31 / 32$ & 15 \\
\hline ETL & 12 & 1 \\
\hline FOV & 16 & 12 \\
\hline SL(mm) & 4 & 2 \\
\hline GAP & 0 & 0 \\
\hline MATRIX & $512 \times 512$ & $320 \times 256$ \\
\hline NEX & 4 & 3 \\
\hline TIME (min) & $5-6$ & $6-7$ \\
\hline Table-1: Imaging parameters for the sequences performed \\
\multicolumn{2}{|r}{ during the MR examination } \\
\hline
\end{tabular}

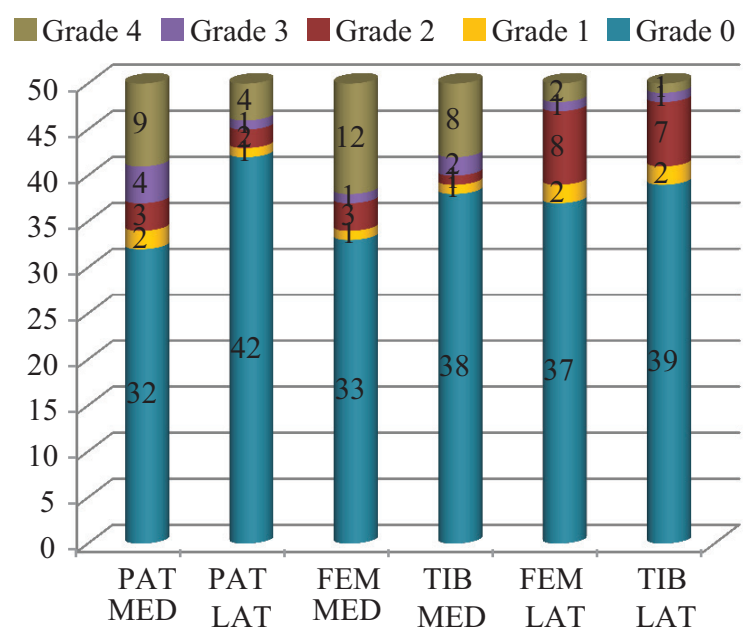

Graph-1: Distribution of Grading of cartilage injury for different articular sufaces of knee on MRI 
(86\%) are below the age of 50 years and 17 cases (14\%) are above 50 years.

Grading and distribution of the cartilage defects: A total of 300 surfaces of 50 knees were evaluated, of which $22(\sim 72 \%)$ were normal surfaces and $79(27 \%)$ showed cartilage defects. $9(\sim 3 \%)$ were grade 1 lesions, $24(\sim 8 \%)$ were grade 2,10 ( 3\%) were grade 3,and 36( 12\%)were grade 4 lesions
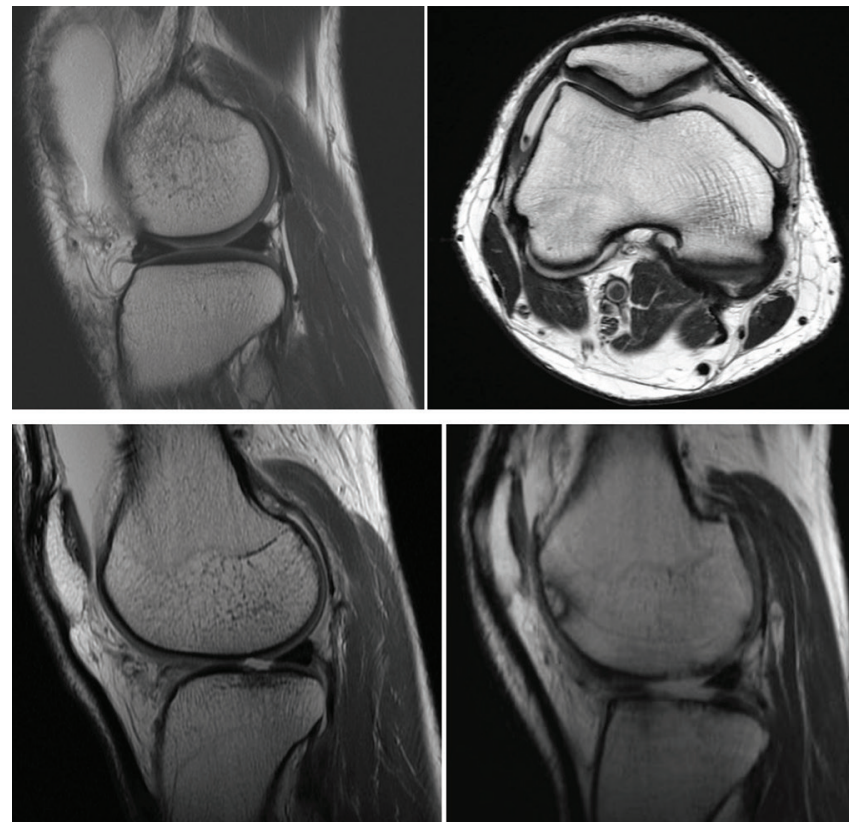

Figure-1: Iw FSE Imaging: Fig A shows the normal tri laminar appearance of cartilage. Fig B shows focal area of increased signal intensity in the retro patellar cartilage without loss of cartilage - G-I cartilage injury. Fig C shows Partial-thickness defect $\geq 50 \%$ involving the lateral tibial condyle with exposure of the normal subchondral bone surface - G -III. Fig D: full thickness cartilage defect with contusion of the subchondral bone. according to arthroscopy. Chondral lesions were seen more frequently in the medial patellar surface cartilage and medial femoral surface cartilage (Table-2, Graph-1).

Sensitivity for iw FSE ranged from as high as $97 \%$ for medial tibial condyle to as low as $62 \%$ for lateral femoral and tibial condyles. Specificity was greater than $90 \%$ and accuracy ranged from $88 \%$ to $96 \%$ respectively.

Sensitivity for 3D SPGR ranged from 98\% for medial tibial condyle to $62 \%$ for lateral femoral and tibial condyles. Specificity was greater than $90 \%$ and accuracy ranged from $88 \%$ to $98 \%$ respectively.
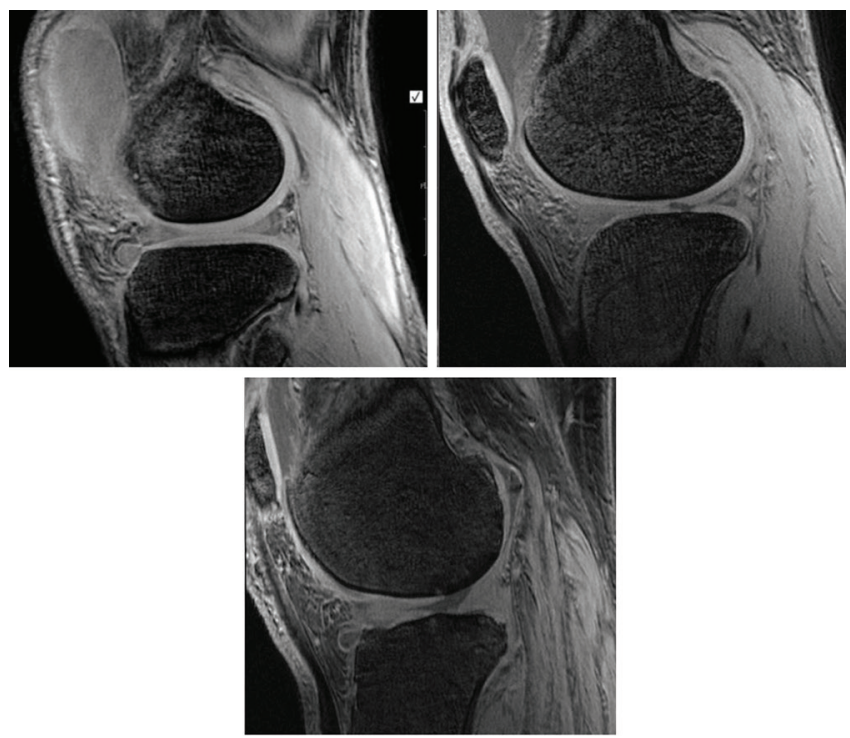

Figure-2: 3DSPGR Imaging: Fig A shows uniform bright appearance of normal cartilage. Fig B shows focal area of reduced signal intensity with Partial-thickness defect $\geq$ $50 \%$ involving the lateral tibial condyle with exposure of the normal subchondral bone surface $-\mathrm{G}-\mathrm{III}$. Fig D: Full thickness cartilage defect with contusion of the subchondral bone-G- IV.

\begin{tabular}{|l|c|c|c|c|c|c|}
\hline Grading & $\begin{array}{c}\text { Patella medial } \\
\text { surface }\end{array}$ & $\begin{array}{c}\text { Patella lateral } \\
\text { surface }\end{array}$ & $\begin{array}{c}\text { Medial Femoral } \\
\text { condyle }\end{array}$ & $\begin{array}{c}\text { Medial Tibial } \\
\text { condyle }\end{array}$ & $\begin{array}{c}\text { Lateral Femoral } \\
\text { condyle }\end{array}$ & $\begin{array}{c}\text { Lateral Tibial } \\
\text { condyle }\end{array}$ \\
\hline Normal/ Grade 0 & 32 & 42 & 33 & 38 & 37 & 39 \\
\hline Grade 1 & 2 & 1 & 1 & 1 & 2 & 2 \\
\hline Grade 2 & 3 & 2 & 3 & 1 & 8 & 7 \\
\hline Grade 3 & 4 & 1 & 1 & 2 & 1 & 1 \\
\hline Grade 4 & 9 & 12 & 8 & 2 & 1 \\
\hline \multicolumn{7}{|l}{} \\
\end{tabular}

\begin{tabular}{|l|c|c|c|}
\hline Iw FSE & Sensitivity\% & Specificity\% & Accuracy\% \\
\hline Hollis G potter et al & 87 & 94 & 92 \\
\hline Hyun-joo Kim et al & 88 & 98 & 96 \\
\hline Our study & $62-96(79)$ & 97 & 92 \\
\hline \multicolumn{2}{|c|}{ Table-3: Comparison of sensitivity, specificity and accuracy of Iw-FSE imaging in our study with similar studies in the literature. } \\
\hline
\end{tabular}

\begin{tabular}{|l|c|c|c|}
\hline 3D SPGR & Sensitivity\% & Specificity\% & Accuracy\% \\
\hline David G Disler et al & $85-95$ & 97 & 84 \\
\hline Lixiao-ming et al & 64 & 87 & 92 \\
\hline Our study & $62-96(79)$ & 96 & 93 \\
\hline \multicolumn{2}{|c|}{ Table-4: Comparison of sensitivity, specificity and accuracy of 3D-SPGR imaging in our study with similar studies in the literature } \\
\hline
\end{tabular}


In our study, $r$ values (spearman's rho correlation) ranged from as high as 0.956 for medial patellar surface cartilage to as low as 0.5 in for lateral tibial surface articular cartilage for both the Intermediate weighted fast spin echo (Iw FSE) and 3D SPGR MR sequences.

\section{DISCUSSION}

The knee joint is one of the most commonly involved joints in external injuries because of the human bipedal nature ${ }^{2,8}$. Injuries include acute, chronic as well as repetitive trauma. The complex structure and function of the articular cartilage can be disrupted by even minor injuries ${ }^{2,9,10}$. Hyaline cartilage defects in the knee are an important source of patient symptoms ${ }^{3,4,11}$. With the recent development of chondrocyte transplantation ${ }^{11,12}$ and other advanced surgical techniques, the pre surgical recognition and characterization of such defects has become increasingly relevant ${ }^{11}$ Detecting hyaline cartilage defects in the knee is important because symptoms and signs associated with such defects can be confused clinically with meniscal tears. Meniscal tears are easily repaired, but treating chondral defects is controversial and of limited prognostic value, because hyaline cartilage does not regenerate but rather repairs with growth of fibrocartilage from sub chondral mesenchyme. Increasing numbers of studies have described the common incidence of hyaline cartilage injuries, their confusion at clinical presentation with meniscal tears, and clinicians' inability to recognize hyaline cartilage injuries with standard MR imaging techniques.

In the study conducted by LI Xiao-ming et al, the incidence of cartilage changes of all the evaluated surfaces was $41 \%$ (339/828), cartilage defects was 22\% (183/828), grade 3 lesions was 3\% (25/828), and grade 4 lesions was 3\% (26/828). Surgical grading of the 324 cartilage surfaces evaluated by Sonin et al shows "normal" (grades 0 and 1) in 241 surfaces (74.4\%); partial-thickness defects (grades 2 and 3) in 56 (17.3\%); and full-thickness defects (grade 4) in 27 surfaces (8.3\%). Of the partial-thickness defects, 29 (9.0\% of the total) were grade 2 and 27 ( $8.3 \%$ of the total) were grade 3 .

In our study, the incidence of cartilage defects in all the evaluated surfaces was $27 \%$ (79/300), grade 1 lesions was $3 \%$, grade 2 lesions was $8 \%$, grade 3 lesions was $3 \%$ and grade 4 lesions was $12 \%$.

The incidences of cartilage lesions of different grades in our study were slightly lower than that of previous studies; however, these differences may be partly because of the relatively smaller sample size as patients with non traumatic knee pain and those with degenerative osteoarthritis were precluded from our research.

As the T2 value of cartilage reflects its collagen content, tissue anisotropy and its water content ${ }^{13,14} \mathrm{Iw}_{\mathrm{w}} \mathrm{FSE}$ imaging can detect early chondromalacia according to the $\mathrm{T} 2$ contrast $^{13,15}$. The magnetization transfer effect is also one of the factors for cartilage contrast on the FSE imaging ${ }^{13,16}$. In addition, subchondral change in the cases of chondral lesions can be detected on the Iw-FSE sequence rather than on the 3D sequence.

Schaefer et al reported that grade I cartilage lesions were underestimated on $3 \mathrm{D}$ sequences and that the intra substantial contrast between chondral lesions and intact cartilage was poor on the 3D sequence. Therefore, the IwFSE images can more easily detect the cartilage lesions ${ }^{13,17}$. The Iw-FSE described in our study offers another advantage in that it allows adjacent menisci, ligaments, and subchondral bone to be evaluated ${ }^{13,18}$ (figure-1).

The pulse sequence described in our study offers a slight advantage in that it allows adjacent menisci, ligaments, and subchondral bone to be evaluated ${ }^{12,19}$. In addition, after arthroscopic treatment, metallic instrumentation (such as suture anchors and compression screws) and residual metallic debris from the arthroscope may cause local disturbance in the magnetic field. Fast-spin-echo sequences are superior to gradient-echo techniques in diminishing the susceptibility artifact caused by metallic instrumentation, which may obscure the overlying articular cartilage $\mathrm{e}^{11,19}$.

The sensitivity, specificity and accuracy of iw-FSE imaging in our study aresimilarto the studies by the Hollis $\mathrm{G}$ potter et al and Hyun-joo Kim et al. The increased sensitivity, specificity and accuracy in our study is due to the adjusted scan parameters like matrix size $(512 \times 512)$, slice thickness ( $4 \mathrm{~mm})$ with no gap, which lead to increased SNR and better detection of cartilage lesions (Table-3).

Sensitivity for 3D SPGR ranged from 98\% for medial tibial condyle to $62 \%$ for lateral femoral and tibial condyles. Specificity was greater than $90 \%$ and accuracy ranged from $88 \%$ to $98 \%$ respectively.

3D SPGR imaging is one of the most commonly used sequences for cartilage evaluation in clinical practice $e^{2,3,4,6,7}$. In our study, chondral lesions of the knee were shown to be accurately detected on 3D SPGR imaging, with sensitivity ranging from $62-96 \%$, specificity of $96 \%$ and accuracy of $93 \%$. Sensitivity, specificity and accuracy of 3D SPGR imaging in our study are almost similar to the studies by David $G$ Disler $^{3,4}$ et al and lixiao-ming ${ }^{2}$ et al (Table-4).

Since fat-suppressed 3DSPGR essentially suppresses all stationary tissue, it is not useful in evaluating the fibrocartilage, ligaments, or soft tissues of the $\mathrm{knee}^{2,20}$ and is not sensitive for marrow edema, which is often an indication for an overlying cartilage defects ${ }^{2}$ (figure-2).

Magnetic resonance imaging demonstrated relatively poor sensitivity with regard to the detection of chondral lesions of the lateral tibial plateau; partly because of the relatively small number of chondral lesions that were noted in this region at arthroscopy. It should be noted, however, that the lateral tibial plateau also was described by Disler et $\mathrm{al}^{3,4}$. as a difficult region in which to detect chondral lesions. This difficulty may also be due to the convex surface of the plateau, which, when subjected to sectioning into tomographic coronal and sagittal images, may impart more partial volume effects and imaging artifacts. In contrast, magnetic resonance imaging demonstrated superior sensitivity ( $>90$ per cent) with regard to the detection of defects involving the patellar facets and medial femoral and tibial condyles which are relatively thick and straight and this finding was consistent with those of other studies.

\section{CONCLUSION}

Hence, our study showed that hyaline cartilage defects of the knee can be accurately identified on cartilage specific 
sequences like intermediate weighted (Iw) FSE and fat suppressed 3D SPGR. Intermediate weighted (Iw) FSE and Fat-suppressed 3D SPGR imaging significantly improved sensitivity for the detection of hyaline cartilage abnormalities over the standard MR imaging techniques we studied

\section{REFERENES}

1. Paavo-Ilari Kuikka, Martti J. Kiuru, Maria H. Niva et al. Sensitivity of Routine 1.0-Tesla Magnetic Resonance Imaging Versus Arthroscopy as Gold Standard in Fresh Traumatic Chondral Lesions of the Knee in Young Adults The Journal of Arthroscopic and Related Surgery 2006;22(10):1033-1039.

2. LI Xiao-ming, Peng Wen-jia, WU Hua, Kacher Daniel, XIA Li-ming, AIFei et al. MRI findings in injured articular cartilage of the knee correlated with surgical findings. Chin Med J 2009;122(21):2624-2630.

3. David G. Disler, Thomas R. McCauley, Chad, Kelman Mark D, Fuchs Lee M, Ratner Carl R Wirth. FatSuppressed Three-Dimensional Spoiled Gradient Echo MR Imaging of Hyaline Cartilage Defects in the Knee: Comparison with Standard MR Imaging and Arthroscopy AJR 1996;61(3):121-132.

4. David G. Disler, Thomas R. McCauley Carl R. Wirth Marc D. Fuchs. Detection of Knee Hyaline Cartilage Defects Using Fat-Suppressed Three-Dimensional Spoiled Gradient-Echo MR Imaging: Comparison with Standard MR Imaging and Correlation with Arthroscopy. AJR:165, August 1995

5. Gonzalo Delgado P. Assessment of articular cartilage using magnetic resonance imaging. Magnetic Resonance Unit, Clinica Avansalud Providencia, Santiago, Chile.

6. Disler DG, McCauley TR, Kelman CG, et al. Fatsuppressed three-dimensional spoiled gradientecho MR imaging of hyaline cartilage defects in the knee: Comparison with standard MR imaging and arthroscopy. AJR Am J Roentgenol 1996; 167(6):127132.

7. David BS, Jeff M, Jean HB, Scott BR. High-resolution 3Dcartilage imaging with IDEAL-SPGR at $3 \mathrm{~T}$. AJR 2007; 189(3):1510-1515.

8. John AC, Mark ES. Imaging of sports-related knee injuries. Radiol Clin N Am 2002; 40(1): 181-202.

9. 17Zhou GD, Wang XY, Miao CL, Liu TY, Zhu L, Liu $\mathrm{DL}$, et al. Repairing porcine knee joint osteochondral defects at non-weight bearing area by autologous BMSC. Natl Med J China (Chin) 2004; 84(5): 925-931.

10. Wa J, Brian JC. Cartilage restoration, part 1: basic science,historical perspective, patient evaluation, and treatment options. Am J Sports Med 2005; 33(1): 295 306.

11. Andrew H. Sonin, Raymond A. Pensy, Michael E. Mulligan Stephen Hatem. Grading Articular Cartilage of the Knee Using Fast Spin-Echo Proton DensityWeighted MR Imaging Without Fat Suppression. AJR 2002;179(4):1159-1166.

12. Hollis G. Potter, James M. Linklater, ANSWORTH a. Allen, Jo A. Hannafin, and Steven b. Haas. Magnetic resonance imaging Of articular cartilage in the knee: An evaluation with use of fast-spin-echo imaging. The journal of bone and joint surgery september 1998;80(9):
1276-1284.

13. Hyun-joo Kim, Sang Hoon Lee, Chang Ho Kang, Jeong Ah Ryu, Myung Jin Shin, Kyung-JaChO, Woo Shin Cho. Evaluation of the Chondromalacia Patella Using a Microscopy Coil: Comparison of the TwoDimensional Fast Spin Echo Techniques and the ThreeDimensional Fast Field Echo Techniques. Korean J Radiol 2011;12(1):78-88.

14. Mosher TJ, Dardzinski BJ. Cartilage MRI T2 relaxation time mapping: overview and applications. Semin Musculoskelet Radiol 2004;8(5):355-368.

15. Mosher TJ, Smith HE, Collins C, Liu Y, Hancy J, Dardzinski BJ, et al. Change in knee cartilage T2 at MR imaging afterrunning: a feasibility study. Radiology 2005;234(6):245-249

16. Yao L, Gentili A, Thomas A. Incidental magnetization transfercontrast in fast spin-echo imaging of cartilage. J Magn Reson Imaging 1996;6(4):180-184

17. Schaefer FK, Kurz B, Schaefer PJ, Fuerst M, Hedderich $\mathrm{J}$, Graessner J, et al. Accuracy and precision in the detectionof articular cartilage lesions using magnetic resonance imaging at 1.5 Tesla in an in vitro study with orthopedic and histopathologic correlation. Acta Radiol 2007;48(2):1131-1137

18. Michel D. Crema, Frank W. Roemer, Monica D. Marra articular cartilage in the knee: current $\mathrm{mr}$ imaging techniques and applications in clinical practice and research RadioGraphics 2011; 31(4):37-62.

19. Potter, H. G.; Rodeo, S. A.; Wickiewicz, T. L.; and Warren, R. F.: MR imaging of meniscal allografts: correlation with clinical and arthroscopic outcomes. Radiology 1996; 198(3): 509-514.

20. Disler DG, Peters TL, Muscoreil SJ, Ratner LM, Wagle WA,Cousins JP, et al. Fat-suppressed spoiled GRASS imaging ofknee hyaline cartilage: technique optimization and comparison with conventional MR imaging. AJR 1994; 163(4): 887-892.

\section{Source of Support: Nil; Conflict of Interest: None}

Submitted: 28-05-2018; Accepted: 30-06-2018; Published online: 10-07-2018 